\title{
1. De la linguistique de la langue à la linguistique du discours, et retour ${ }^{1}$
}

Patrick Charaudeau

Université Paris XIII

\section{Introduction}

Le terme de linguistique est devenu ambigu. À quelle notion renvoie-til ? En ses débuts, avec l'arrivée du structuralisme, il était réservé aux études descriptives et non prescriptives de la langue, s'opposant ainsi à la grammaire, dite traditionnelle. La linguistique était alors la discipline qui analysait les systèmes phonologique, morphologique, syntaxique et sémantique des langues. Et puis le domaine s'est élargi aux aspects sociologiques (sociolinguistique), psychologique (psycholinguistique), ethnologique (ethnolinguistique) de la langue, et à ses divers usages : communicationnel, conversationnel, ethnographique, engendrant par là même des courants disciplinaires tels la sémiotique et l'analyse des discours.

Cela est vite dit et ne tient pas compte de la multiplicité des théories et des méthodologies qui sont nées pour tenter de rendre compte de ces différents aspects et de leur combinaison. Mais il ressort de cet ensemble quelque peu hétérogène deux grandes tendances du point de vue de la constitution de l'objet et de la démarche d'analyse. L'une qui est centrée sur l'étude des systèmes des langues dans leurs diverses dimensions comme on vient de le dire, l'autre davantage centrée sur les usages, le langage en tant qu'acte et ses procédés de mise en scène. Ces deux tendances ne sont pas exclusives l'une de l'autre ; on verra comment elles s'articulent, mais en leur fondement théorico-méthodologique elles se différencient suffisamment pour que l'on puisse distinguer - ce que j'ai proposé dans d'autres écrits - une linguistique de la langue et une linguistique du discours.

Comment citer ce chapitre :

Charaudeau, Patrick, De la linguistique de la langue à la linguistique du discours, et retour. In: Engwall, Gunnel \& Fant, Lars (eds.) Festival Romanistica. Contribuciones lingüisticas - Contributions linguistiques - Contributi linguistici - Contribuições linguisticas. Stockholm Studies in Romance Languages. Stockholm: Stockholm University Press. 20I 5, pp. 3-I2. DOI: http://dx.doi.org/Io.I6993/bac.a. License: CC-BY 
C'est en me plaçant du point de vue sémantique que je voudrais montrer ce qui justifie cette distinction. Car de ce point de vue, on est obligé de s'interroger non seulement sur le sens des mots, en soi, mais aussi sur le sens qu'ils transmettent. Roland Barthes, toujours aussi pertinent, a rappelé que si le signe " signifie », on oublie qu'il "signifie à »². En effet, le phénomène de signifiance résulte de ces deux orientations : une orientation centripète qui tend à stabiliser le sens sur lui-même, une orientation centrifuge qui tend à construire du sens en fonction de ses conditions d'emploi dans des actes de communication. On peut dire que le langage signifie en même temps qu'il transmet du sens, que c'est dans l'acte même de transmission qu'il signifie avec une intention de produire un certain effet sur l'autre du langage. Interrogeons-nous donc sur ce double sens qu'on dira de langue et de discours.

\section{Le sens de langue n'est pas le sens de discours}

Soit l'énoncé : «J'ai trente ans ». Une analyse sémantique, hors contexte, permettrait de montrer qu'il s'agit d'une assertion dans laquelle à un certain actant (Je) est attribuée (avoir) une certaine propriété (ans), laquelle est quantifiée (trente), le tout dans un acte d'énonciation qui dit que cette assertion doit être rapportée au sujet parlant lui-même ( $\mathrm{Je}$ ) dans une modalisation élocutive d'affirmation. Si, de plus, on consulte un dictionnaire, on apprendra que ce " ans » désigne une certaine segmentation du temps, et que, combiné à une certaine quantification (de un à cent), il peut désigner un âge de la vie.

Si maintenant on considère cet énoncé en contexte communicationnel, émanant d'un certain locuteur, comme une réplique à une assertion antérieure. Par exemple, imaginons qu'un locuteur conversant avec un ami qui s'étonne de le voir se retirer de la compétition sportive, réplique : "J'ai trente ans »; alors cet énoncé signifiera : 'Je suis trop vieux'. Cela suppose évidemment que le locuteur en question soit un sportif et que l'interlocuteur le sache. Imaginons maintenant qu'il s'agit d'une personne qui vient d'être licenciée d'une entreprise, qu'elle en informe un de ses amis et que celui-ci tente une explication : "C'est peut-être parce que tu as passé l'âge ? »; alors l'énoncé « J'ai trente ans » signifiera quelque chose comme : 'Pourtant, je suis encore jeune'.

Dès lors, on peut se poser la question de savoir quelle grammaire et quel dictionnaire pourraient dire que cet énoncé signifie 'vieux' ou 'jeune' ? Une chose est ce que signifient les mots en langue, autre chose ce qu'ils signifient en contexte communicationnel, lequel détermine 
l'enjeu de l'acte de langage. C'est que l'enjeu de l'acte de langage ne se trouve pas tant dans l'explicite de ce qui est dit (la langue) que dans l'implicite qu'il véhicule, la combinaison des deux fabriquants du discours. Tout acte de langage a, de façon constitutive, une double dimension explicite et implicite, indissociable l'une de l'autre.

\section{Deux conceptualisations du signe}

De cette observation, on peut tirer un certain nombre de conséquences quant à la façon de concevoir ce que serait la conceptualisation du signe linguistique:

- le signe de langue, d'après une tradition maintenant bien établie, se définit selon une triple dimension : structurelle, car il s'informe et se sémantise de façon systémique au croisement des co-occurrences syntagmatiques (combinaison de trente et ans) et des oppositions paradigmatiques ( $\mathrm{Je}$ n'est pas $\mathrm{Tu}$, an n'est pas mois, et réciproquement); contextuelle, dans la mesure où il est investi de sens par un contexte linguistique qui doit assurer une certaine isotopie ; référentielle dans la mesure ou tout signe réfère à une réalité du monde dont il construit la signifiance.

- le signe de discours, lui, se définit selon une double dimension : situationnelle et énonciative, car il dépend pour son sens des composantes de la situation de communication et d'un certain processus d'énonciation dans lesquels il apparaît ; interdiscursive (ou intertextuelle) $)^{3}$, car son sens dépend également des discours déjà produits qui constituent des domaines de savoir normés.

Cela explique que le signe de langue soit répertoriable, catégorisable, et que son sens relève du probable, parce que parmi un ensemble de sens possibles, au vu des combinaisons syntagmatiques et des oppositions paradigmatiques, tout récepteur ou observateur entendra, probablement, la même chose. Par exemple : étant donné les sens possibles de an, sa quantification, son attribution à une personne, on s'entendra sur le sens probable de : 'âge', ce que pourra confirmer le dictionnaire, comme l'un des sens possibles. Le signe de discours, lui, n'est pas catégorisable car il est toujours dépendant d'autre chose que de lui-même, d'un quelque chose d'externe à l'énoncé, son sens relevant du plausible. Par exemple : étant donné que c'est un sportif de haut niveau qui parle en justifiant son retrait de cette activité, étant donné un savoir sur la 
limite d'âge des sportifs au regard de la compétition, on peut en inférer, plausiblement : 'il est trop vieux', et dans ce cas, le dictionnaire ne sera d'aucun recours. De même, si un mot comme « intellectuel », dans un énoncé du genre : "C'est un intellectuel ! ", peut prendre tantôt une valeur positive, tantôt une valeur négative, c'est parce que circulent dans les groupes sociaux des discours qui, soit, opposent les intellectuels aux sportifs ou aux gens qui savent s'engager physiquement dans des actions (valeur négative), soit les opposent à ceux qui n'agissent que par pulsion, sans se contrôler, sans réfléchir (valeur positive).

On voit donc que le signe fait l'objet d'une double conceptualisation: une conceptualisation linguistique (de la langue) qui se fait dans un double mouvement de sémantisation entre l'universel et le particulier, le particulier et l'universel, à des niveaux plus ou moins abstraits, comme le montrent les travaux des sémanticiens et les théories des prototypes et des topoï ${ }^{4}$; une conceptualisation discursive (du discours) qui se fait dans un double mouvement de sémantisation entre une norme sociale et une spécificité individuelle de savoir, le savoir étant ici conçu comme un ensemble de systèmes de connaissance et de croyance partagées. On voit que pour déterminer le sens de discours il faut avoir recours à du contexte. Mais, qu'est-ce que le contexte?

\section{De la diversité des contextes}

Voilà encore une notion passe-partout. Lorsqu'elle est définie, c'est tantôt de façon générale (tout est contexte), tantôt de façon restrictive, comme l'ensemble des co-occurrents qui environne physiquement une unité linguistique ; tantôt elle est confondue avec la situation de communication (le contexte d'une conférence), tantôt elle désigne plus amplement la dimension culturelle d'un phénomène (le contexte américain ou le contexte français).

Si l'on adopte le point de vue du sujet parlant dans son travail de production du langage, on peut supposer que pour réaliser son acte de langage, il tient compte, à la fois, de l'environnement linguistique immédiat de l'énoncé, de la situation d'énonciation et des discours déjà produits. Si l'on adopte le point de vue du récepteur dans son travail d'interprétation, il est aisé de constater qu'il doit avoir recours à plusieurs types de contexte : un contexte linguistique constitué des co-occurrents permettant de déterminer le sens premier des mots ; un contexte textuel constitué de textes produits par une même source (par exemple, les divers écrits d'un même auteur) qui permet par exemple de 
saisir ce que signifie le mot " œil » chez Baudelaire ; un contexte paratextuel constitué de textes ou fragments de textes se trouvant en coprésence dans un même espace scriptural (les titres, sous-titres, chapeaux, légendes d'une page de journal) ; un contexte hypertextuel', constitué de textes qui se citent, se renvoient les uns aux autres, se reprennent et se transforment, comme sont les pastiches et parodies ; un contexte intertextuel (ou interdiscursif), constitué de textes et discours qui circulent dans l'espace social, et qui sont convoqués par le sujet récepteur pour justifier ses interprétations.

À ces différents types de contexte, il faut ajouter un contexte qui tient également compte d'un " hors-texte »(parfois appelé cotexte), c'est-à-dire des données présentes dans les conditions de production de l'acte de langage : le contexte situationnel'. Il s'agit, ici, de la situation de communication qui nous oblige (que l'on soit en position d'interlocuteur, de lecteur ou même d'analyste) à nous interroger sur l'identité de celui qui parle (un sportif, un travailleur), sur la finalité de l'échange (informer) qui détermine l'enjeu de signification de l'échange langagier, sur le dispositif et les circonstances matérielles (conversation) de celui-ci. Cet ensemble de composantes constitue les conditions de production du discours.

\section{Les trois dimensions de l'acte de langage}

Cette observation conduit à avancer que l'activité langagière des êtres parlants s'appuie à la fois sur une mémoire linguistique (l'organisation des systèmes), une mémoire interdiscursive (les savoirs supposément partagés) et une mémoire situationnelle (les conditions psychologiques et sociologiques de l'acte de communication). Des mouvements centripète et centrifuge, à l'articulation desquels se construit le sens qui consiste pour les sujets à parler du monde à travers leur relation à l'autre. Aussi peut-on dire que l'acte de langage comprend trois dimensions :

I. une dimension topicalisante où se construisent les savoirs sur le monde : savoirs de connaissance et savoirs de croyance qui constituent les "imaginaires sociaux » qui caractérisent une société. C'est-à-dire la façon dont les individus vivant en société se représentent le monde et jugent les comportements humains, organisant ces savoirs en doctrines, idéologies ou tout simplement opinions plus ou moins stéréotypées. C'est ce phénomène de circulation et d'entrecroisement des paroles qui s'échangent 
dans l'espace public ou interpersonnel, qui est appelé, parfois, " intertextualité » (Genette) $)^{7}$, parfois, « dialogisme » $(\text { Bakhtine })^{8}$, parfois, « interdiscursivité ».

2. une dimension énonciative où se joue la mise en scène discursive avec son organisation descriptive, narrative, argumentative, énonciative et le choix des mots du lexique qui l'accompagne. Cependant, il convient ici de distinguer l'énonciation du point de vue de la langue et du point de vue du discours, bien que les deux soient intimement liés ${ }^{9}$. Du point de vue de la langue, l'énonciation a été définie par les textes, considérés comme fondateurs, d'Emile Benveniste ${ }^{\text {Io }}$ qui pose la présence des sujets $J e$ et Tu comme déterminant l'acte même de langage, dans la mesure où parler, c'est toujours, pour un locuteur Je, s'adresser à un interlocuteur $T u$, lequel peut à son tour prendre possession de la parole. Il s'établit ainsi entre eux un rapport de réciprocité non symétrique : pas de $J e$ sans $T u$, pas de Tu sans $J e$. À partir de ce principe de fonctionnement du langage, - qui détermine la présence de "l'homme dans la langue »-Benveniste décrit ce qu'il appelle l'appareil formel de l'énonciation ${ }^{\text {II }}$, c'est-à-dire l'ensemble des formes et systèmes linguistiques qui expriment d'une façon ou d'une autre les différents positionnements du sujet parlant en relation avec son interlocuteur et ce qu'il dit : les pronoms personnels de $\mathrm{I}^{\text {ère }}$ et $2^{\text {ème }}$ personne (positionnement des locuteurs), les temps des verbes et les adverbes de temps (positionnement dans le temps), les déictiques (positionnement par rapport à l'espace), les verbes, les adverbes de modalité et le discours rapporté (positionnement par rapport à l'énoncé), enfin, des adjectifs affectifs (positionnement par rapport à la subjectivité du locuteur). Du point de vue du discours, l'énonciation englobe la totalité de l'acte de langage. C'est le processus par lequel un sujet parlant met en scène son dire, l'ordonnant en fonction de divers paramètres lui permettant de construire une description, un récit, une argumentation, etc. Il s'agit là de deux définitions de l'énonciation dont on voit cependant comment elles s'articulent, la conception discursive déterminant la mise en scène de l'acte de langage, mais à l'aide de la conception linguistique qui fournit au sujet parlant l'appareil des marques linguistiques avec lesquelles il pourra s'exprimer.

3. une dimension communicationnelle qui oblige à prendre en compte l'aspect externe de l'acte de langage, là où se trouvent 
ses conditions psycho-sociales de production et d'interprétation. On ne peut parler, écrire ni interpréter si on ne tient pas compte de ces prédéterminations. C'est ce qui fait qu'une même phrase n'aura pas le même sens et ne produira pas le même effet selon la situation dans laquelle elle a été produite : qui parle à qui (identité), avec quel enjeu (finalité), dans quel dispositif (genre). Cet ensemble de conditions de production/interprétation situationnelles constitue un contrat de communication, lieu de contraintes, qui donne des instructions discursives au sujet parlant, contraintes qu'il devra suivre pour procéder à son acte d'énonciation, et dont le sujet interlocuteur devra tenir compte pour interpréter. La situation de communication ne se confond donc pas avec la situation d'énonciation : la première concerne les conditions externes de production (englobante), la seconde, la mise en scène discursive (spécifiante), en fonction des instructions données par la première, et en ayant recours à l'appareil de l'énonciation que lui fournit le système linguistique.

\section{Le sens se construit par inférence}

Le sens se construit à l'articulation de ces trois dimensions, ce qui veut dire qu'il n'est pas ce qui émane du seul énoncé, mais de celui-ci inscrit dans un ensemble de conditions de production. De plus, la seule intention du sujet parlant n'est pas non plus ce qui constitue la totalité du sens, car il y a toujours un sujet interprétant qui lui, aussi, construit du sens, et c'est donc de la rencontre entre les deux partenaires de l'échange langagier dans une situation donnée que surgit un ensemble de sens possibles. Le sens d'un acte de langage, quel qu'il soit, est toujours le résultat d'une co-construction.

Une phrase comme "Les noirs sont différents des blancs » n'est pas raciste en soi. Elle le sera si nous savons : qui la prononce (par exemple, un acteur politique d'extrême droite, dont les discours sont explicitement racistes) ; dans quelle dispositif (par exemple, une interview journalistique, lors d'une campagne électorale); dans quelle mise en scène énonciative (une constatation empirique qui se veut d'évidence, pour justifier une discrimination sociale). Mais cette même phrase prononcée par un ethnologue qui est en train de décrire le statut qu'occupent les individus dans une population métissée, n'aura pas un effet de sens raciste.

$\mathrm{Du}$ coup, on voit que la production de sens, qu'elle provienne d'un sujet locuteur ou d'un sujet récepteur, résulte d'une activité 
inférentielle, non nécessairement prévisible, parce qu'elle s'appuie sur divers contextes. Et selon le type de contexte auquel on se réfère, on aura affaire à des inférences contextuelles, situationnelles ou interdiscursives, ce que représente le schéma suivant :

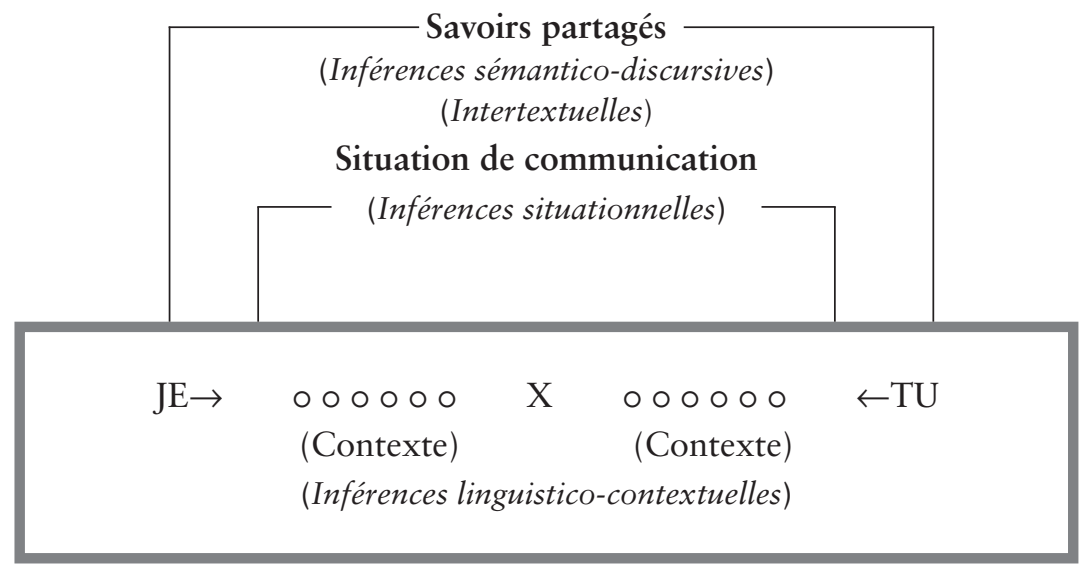

Le sens de discours n'est donc pas, comme on a voulu le dire à un moment donné, une transposition du sens de la phrase dans un au-delà de celle-ci. Car le sens de la phrase est de l'ordre de la prédication, alors que le sens du discours est de l'ordre de la problématisation. Sens de langue et sens de discours ne suivent pas les mêmes procédures de calcul : le premier s'obtient par un calcul déductif de probabilité, le second, par calcul d'inférence plausible selon les trois types ci-dessus décrits.

\section{Du sens de langue au sens de discours via l'énonciation}

Après avoir distingué ces deux types de signe, ces deux procédés de calcul du sens, on pourrait se demander si il n'existe pas tout de même un lien entre les deux, car on peut aussi défendre l'idée que le langage est un tout qui dans ses différentes réalisations tisse une toile sémantique, parfois labyrinthique, dont chaque fil est lié de façon plus ou moins directe aux autres. Ce lien, pour moi, se trouve dans l'énonciation, dans le procédé de mise en scène énonciative. Car c'est par lui que s'intriquent les sens de langue et de discours. À force d'intrication, ce qui dépend de l'aléa des contextes finit par s'inscrire dans la langue, et le sens de discours plausible, spécifique d'une situation particulière, par récurrence, s'ajoute aux autres traits sémantiques du signe en constituant par sédimentation une potentialité de sens, ensuite disponible pour d'autres emplois. C'est ainsi qu'évoluent les langues. Si « 30 ans » 
peut signifier 'jeune' ou 'vieux', si « intellectuel » peut signifier une qualité positive ou négative, ce n'est pas que ces termes soient directement porteurs de ces sens (une fois de plus on ne pourrait les répertorier dans un dictionnaire), c'est que ces mots ont dans leur sémantisme quelque chose (un ou des traits) qui - sans être explicite - est potentiellement disponible, ce qui leur donne la capacité d' " accueillir» des sens non prévus qui sont apportés par le contexte interdiscursif dont j'ai parlé. C'est aussi cette virtualité qui permet d'expliquer l'évolution du sens des mots. Comment est-on passé du « purros » grec qui signifiait 'roux' et 'feu' au latin « burra » qui signifiait 'étoffe de longs poils' à " robe de bure », « bourrelet » et " bourreau », si ce n'est par ce jeu de virtualités successives qui ont accueilli du sens qui n'était pas prévu à chacun de ces stades et qui s'est construit dans l'interdiscursivité ?

C'est donc bien par le biais du processus d'énonciation que s'établit un lien potentiel entre sens de langue et sens de discours, à condition d'admettre cependant que cela ne se fait pas nécessairement par continuité. C'est plutôt que le sens de discours arrivant par les savoirs qui se construisent dans la pratique sociale, il se trouve ensuite comme 'inoculé' dans le sens de langue qui après quelques hésitations finit par l'accepter, voire l'intégrer au point, peut-être, de se l'approprier et de le catégoriser dans une nouvelle dénotation.

Pour en revenir à la proposition de départ, on voit au terme de cette démonstration, qu'une linguistique du discours présuppose une linguistique de la langue : la première ne peut exister sans la seconde. Mais en retour, il faut accepter de considérer qu'une linguistique de la langue ne peut, à elle seule, prétendre rendre compte du sens des actes de langage produits en situation de communication réelle : elle a besoin d'être complétée par une linguistique du discours. Dans un cas comme dans l'autre, il s'agit de " traquer " le sens. Une double traque, à travers un calcul d'ordre probabiliste et d'ordre inférentiel, non nécessairement prévisible, sur un même terreau de virtualités de sens, et toujours dans un mouvement de va-et-vient entre le général et le particulier.

\section{Notes}

I. Ayant été amené à développer ce point de vue dans d'autres colloques, on retrouvera ici des parties d'une même démonstration. 
2. Roland Barthes par roland barthes, Seuil, coll. "Écrivains de toujours", Paris, 1975 .

3. Ici, nous ne ferons pas de distinction entre ces deux notions.

4. Voir Martin (I99I) et Anscombre \& Ducrot (1983).

5. Les contextes paratextuel, métatextuel et hypertextuel sont les propositions de Genette (1982).

6. Parfois appelé « contexte communicationnel ».

7. Genette (1982).

8. Bakhtine (I98I).

9. Voir Dictionnaire d'Analyse du Discours (2002).

ıo. Voir Benveniste, Émile (I966), particulièrement le chapitre intitulé "L'homme dans la langue ».

I I. Benveniste (I970).

\section{Références}

Anscombre, Jean-Claude \& Oswald Ducrot. 1983. L'argumentation dans la langue. Liège : Mardaga.

Bakhtine, Mikhail. I98 I. «Écrits du cercle de Bakhtine ». In : Tzvetan Todorov. Mikhail Bakbtine, le principe dialogique. Paris : Seuil.

Barthes, Roland. 1975. Roland Barthes par roland barthes, Paris : Seuil, coll. «Écrivains de toujours ».

Benveniste, Émile. I970. «L'appareil formel de l'énonciation ». Langages, I7. I 2-I 8 .

Benveniste, Émile. 1966. Problèmes de linguistique générale. Paris : Gallimard.

Charaudeau Patrick \& Dominique Maingueneau. 2002. Dictionnaire d'Analyse du Discours. Paris : Seuil.

Genette, Gérard. 1982. Palimpsestes. Paris : Seuil, coll. « Poétique ».

Martin, Robert. I99I. "Typicité et sens des mots ». In : Dubois, Danièle (dir.). Sémantique et cognition. Catégories, prototypes, typicalité. Paris : Éditions du CNRS. I 5 I-I 59. 